\title{
A NOTE ON SURFACES IN THE NORMAL BUNDLE OF A CURVE
}

\author{
Doohann LeE* and HeungSu YI**
}

\begin{abstract}
In 3-dimensional Euclidean space, the geometric figures of a regular curve are completely determined by the curvature function and the torsion function of the curve, and surfaces are the fundamental curved spaces for pioneering study in modern geometry as well as in classical differential geometry. In this paper, we define parametrizations for surface by using parametric functions whose images are in the normal plane of each point on a given curve, and then obtain some results relating the Gaussian curvature of the surface with curvature and torsion of the given curve. In particular, we find some conditions for the surface to have either nonpositive Gaussian curvature or nonnegative Gaussian curvature.
\end{abstract}

\section{Introduction}

In 3-dimensional Euclidean space $\mathbb{R}^{3}$, the geometric figures of a regular curve are completely determined by the curvature function and the torsion function of the curve, and geometric properties of some special curves are studied until quite recently [3],[4],[5]. Also, intrinsic surfaces are the fundamental curved spaces for pioneering study in modern geometry as well as in classical differential geometry [1],[2].

In differential geometry, a 2-dimensional differentiable manifold or simply a surface $M$ is defined by locally assigning several overlapping parametrizations which cover some open neighborhood of each point of $M$, and the Gaussian curvature $K(p)$ of $p \in M$ can be extrinsically calculated by means of the differentiable unit normal vector field $\mathbf{n}[6],[7]$.

Received January 07, 2014; Accepted April 07, 2014.

2010 Mathematics Subject Classification: Primary 53A05, 53A04.

Key words and phrases: curve, surface, Gaussian curvature.

*This work was supported by the Gachon University research fund of 2014. (2011).

**This study is supported by the Research Grant of Kwangwoon University

Correspondence should be addressed to Doohann Lee, dhl221@gachon.ac.kr. 
In this paper, we define parametrizations for surface by using parametric functions whose images are in the normal plane of each point on a given curve, and then obtain some results relating the Gaussian curvature of the surface with curvature and torsion of the given curve. In particular, we find some conditions for the surface to have either nonpositive Gaussian curvature or nonnegative Gaussian curvature.

\section{Curvatures of surfaces in the normal bundle of a curve}

Let $\gamma$ be a regular curve with a unit speed in $\mathbb{R}^{3}$. We assume that $\gamma(s)$ is of class $C^{3}$ defined on an open interval $I$, and use familiar notations $\kappa, \tau, \mathbf{T}, \mathbf{N}, \mathbf{B}$ for curvature, torsion, tangent vector field, principal normal vector field, binormal vector field of $\gamma$, respectively. Throughout this paper we use the following Frenet formulas:

$$
\frac{d \mathbf{T}}{d s}=\kappa \mathbf{N}, \quad \frac{d \mathbf{N}}{d s}=-\kappa \mathbf{T}+\tau \mathbf{B}, \quad \frac{d \mathbf{B}}{d s}=-\tau \mathbf{N} .
$$

Consider a parametrization $\mathbf{x}$ on an open rectangle $I \times J\left(\subset \mathbb{R}^{2}\right)$ defined by

$$
\mathbf{x}(s, t)=\gamma(s)+f(s, t) \mathbf{N}(s)+g(s, t) \mathbf{B}(s),
$$

where $f(s, t), g(s, t)$ are smooth real-valued functions defined on $I \times J$.

DeFINITION 2.1. We call the parametrization $\mathbf{x}$ defined as above by the normal sectional parametrization of $\gamma$.

For simplicity of notations, we will abbreviate the variables $s$ and $t$ of scalar functions and vector fields, and denote the partial derivative $\frac{\partial f}{\partial s}$ by $f_{s}$. Then, by the Frenet formulas, we obtain the basic equations as follows:

Remark 2.2. Let $R(s, t)=1-\kappa f$ and $S(s, t)=f_{s} g_{t}-f_{t} g_{s}$. Then, we have

(1) $\mathbf{x}_{s}=R \mathbf{T}+\left(f_{s}-\tau g\right) \mathbf{N}+\left(g_{s}+\tau f\right) \mathbf{B}$.

(2) $\mathbf{x}_{t}=f_{t} \mathbf{N}+g_{t} \mathbf{B}$.

(3) $\mathbf{x}_{s} \times \mathbf{x}_{t}=\left[S-\tau\left(f f_{t}+g g_{t}\right)\right] \mathbf{T}-R g_{t} \mathbf{N}+R f_{t} \mathbf{B}$.

The intrinsic properties of surfaces are determined by the first fundamental form on the tangent space at each point. We use the following classical notations:

$$
E=\mathbf{x}_{s} \cdot \mathbf{x}_{s}, F=\mathbf{x}_{s} \cdot \mathbf{x}_{t}, G=\mathbf{x}_{t} \cdot \mathbf{x}_{t}, g=E G-F^{2} .
$$


Proposition 2.3. Let $\mathbf{x}$ be a normal sectional parametrization of $\gamma$ defined as above. Then, the coefficients of the metric tensor are as follows:

(1) $E=R^{2}+\left(f_{s}^{2}+g_{s}^{2}\right)+2 \tau\left(f g_{s}-g f_{s}\right)+\tau^{2}\left(f^{2}+g^{2}\right)$.

(2) $F=\left(f_{s} f_{t}+g_{s} g_{t}\right)+\tau\left(f g_{t}-g f_{t}\right)$.

(3) $G=f_{t}^{2}+g_{t}^{2}$.

(4) $g=\left\|\mathbf{x}_{s} \times \mathbf{x}_{t}\right\|^{2}=\left[S-\tau\left(f f_{t}+g g_{t}\right)\right]^{2}+R^{2}\left(f_{t}^{2}+g_{t}^{2}\right)$.

Theorem 2.4. Assume that $\gamma$ is a unit speed plane curve. Let $p$ be a point in the image $\operatorname{Im}(\mathbf{x})$ of the normal sectional parametrization $\mathbf{x}$ given in Definition 2.1. Then the Gaussian curvature $K$ at $p$ is

$$
\begin{aligned}
K(p) & =-\frac{1}{g^{2}}\left[\kappa g_{t}\left(f_{t} g_{t t}-g_{t} f_{t t}\right) R^{3}+\kappa^{2} f_{t}^{2} S^{2}\right] \\
& +\frac{R S}{g^{2}}\left[-\left(2 \kappa f_{s}+\kappa_{s} f\right)\left(f_{t} g_{t t}-g_{t} f_{t t}\right)+2 \kappa f_{t}\left(f_{t} g_{s t}-g_{t} f_{s t}\right)\right] \\
& +\frac{R^{2}}{g^{2}}\left[\left(f_{t} g_{s s}-g_{t} f_{s s}\right)\left(f_{t} g_{t t}-g_{t} f_{t t}\right)-\left(f_{t} g_{s t}-g_{t} f_{s t}\right)^{2}\right] .
\end{aligned}
$$

Proof. From the condition for a plane curve $\gamma$ and the above proposition, we have

(1) $E=R^{2}+\left(f_{s}^{2}+g_{s}^{2}\right)$,

(2) $F=\left(f_{s} f_{t}+g_{s} g_{t}\right)$,

(3) $G=f_{t}^{2}+g_{t}^{2}$,

(4) $g=S^{2}+R^{2}\left(f_{t}^{2}+g_{t}^{2}\right)$.

Also, by (3) in Remark 2.2, the unit normal vector field $\mathbf{n}$ is

$$
\mathbf{n}=\frac{1}{\sqrt{g}}\left[S \mathbf{T}-R g_{t} \mathbf{N}+R f_{t} \mathbf{B}\right]
$$

By differentiating $\mathbf{x}_{s}=R \mathbf{T}+f_{s} \mathbf{N}+g_{s} \mathbf{B}$ and $\mathbf{x}_{t}=f_{t} \mathbf{N}+g_{t} \mathbf{B}$, we obtain that

$$
\begin{gathered}
\mathbf{x}_{s s}=\left(-\kappa_{s} f-2 \kappa f_{s}\right) \mathbf{T}+\left(\kappa R+f_{s s}\right) \mathbf{N}+g_{s s} \mathbf{B}, \\
\mathbf{x}_{s t}=\left(-\kappa f_{t}\right) \mathbf{T}+f_{s t} \mathbf{N}+g_{s t} \mathbf{B},
\end{gathered}
$$

and

$$
\mathbf{x}_{t t}=f_{t t} \mathbf{N}+g_{t t} \mathbf{B}
$$

Hence, we obtain the coefficients of the second fundamental form as follows:

$$
\begin{aligned}
L=\mathbf{x}_{s s} \cdot \mathbf{n} & =\frac{1}{\sqrt{g}}\left[S\left(-\kappa_{s} f-2 \kappa f_{s}\right)-R\left(\kappa g_{t} R+f_{s s} g_{t}-f_{t} g_{s s}\right)\right], \\
M & =\mathbf{x}_{s t} \cdot \mathbf{n}=\frac{1}{\sqrt{g}}\left[-\kappa f_{t} S+R\left(f_{t} g_{s t}-f_{s t} g_{t}\right)\right],
\end{aligned}
$$


and

$$
N=\mathbf{x}_{t t} \cdot \mathbf{n}=\frac{R}{\sqrt{g}}\left(f_{t} g_{t t}-f_{t t} g_{t}\right)
$$

Therefore,

$$
\begin{aligned}
L N-M^{2}= & -\frac{1}{g}\left[\kappa g_{t}\left(f_{t} g_{t t}-g_{t} f_{t t}\right) R^{3}+\kappa^{2} f_{t}^{2} S^{2}\right] \\
& +\frac{R S}{g}\left[-\left(2 \kappa f_{s}+\kappa_{s} f\right)\left(f_{t} g_{t t}-g_{t} f_{t t}\right)+2 \kappa f_{t}\left(f_{t} g_{s t}-g_{t} f_{s t}\right)\right] \\
& +\frac{R^{2}}{g}\left[\left(f_{t} g_{s s}-g_{t} f_{s s}\right)\left(f_{t} g_{t t}-g_{t} f_{t t}\right)-\left(f_{t} g_{s t}-g_{t} f_{s t}\right)^{2}\right] .
\end{aligned}
$$

Since $K=\frac{L N-M^{2}}{g}$, we obtain the desired result.

As a consequence of the theorem, we obtain the following corollary.

Corollary 2.5. If $\gamma$ is a unit speed straight line, then

$$
K(p)=\frac{\left(f_{t} g_{s s}-g_{t} f_{s s}\right)\left(f_{t} g_{t t}-g_{t} f_{t t}\right)-\left(f_{t} g_{s t}-g_{t} f_{s t}\right)^{2}}{\left(S^{2}+f_{t}^{2}+g_{t}^{2}\right)^{2}} .
$$

Proof. Since any straight line has the constant curvature $\kappa=0$, we have $R=1$.

Proposition 2.6. Assume that $\gamma$ is a unit speed space curve. If the parametrization $\mathbf{x}$ is defined by

$$
\mathbf{x}(s, t)=\gamma(s)+f(s, t) \mathbf{N}(s)+t \mathbf{B}(s),
$$

that is, $g(s, t)=t$, then the coefficients of the second fundamental form of $\mathbf{x}$ are as follows:

$$
\begin{gathered}
L=\quad \frac{1}{\sqrt{g}}\left[\left(f_{s}-\tau\left(f f_{t}+t\right)\right)\left(R_{s}+\kappa\left(t \tau-f_{s}\right)\right)\right. \\
\left.\quad-R\left(\kappa R+f_{s s}-t \tau_{s}-\tau^{2} f\right)+f_{t} R\left(2 \tau f_{s}-t \tau^{2}+\tau_{s} f\right)\right], \\
M=\frac{1}{\sqrt{g}}\left[-f_{s} f_{t}+\tau f_{t}\left(f f_{t}+t\right)+R\left(\tau+\tau f_{t}^{2}-f_{s t}\right)\right],
\end{gathered}
$$

and

$$
N=\frac{-f_{t t} R}{\sqrt{g}},
$$

where $g=\left[f_{s}-\tau\left(f f_{t}+t\right)\right]^{2}+R^{2}\left(1+f_{t}^{2}\right)$. 
Proof. At first, we note that $S(s, t)=f_{s}(s, t)$. By Remark 2.2, we have

$$
\mathbf{x}_{s}=R \mathbf{T}+\left(f_{s}-t \tau\right) \mathbf{N}+(f \tau) \mathbf{B}, \quad \mathbf{x}_{t}=f_{t} \mathbf{N}+\mathbf{B},
$$

and

$$
\mathbf{x}_{s} \times \mathbf{x}_{t}=\left[f_{s}-\tau\left(f f_{t}+t\right)\right] \mathbf{T}-R \mathbf{N}+R f_{t} \mathbf{B} .
$$

Also, since

$$
\begin{gathered}
\mathbf{x}_{s s}=\left(R_{s}+\kappa\left(t \tau-f_{s}\right)\right) \mathbf{T}+\left(\kappa R+f_{s s}-t \tau_{s}-\tau^{2} f\right) \mathbf{N}+\left(2 \tau f_{s}-t \tau^{2}+\tau_{s} f\right) \mathbf{B}, \\
\mathbf{x}_{s t}=-f_{t} \mathbf{T}+\left(f_{s t}-\tau\right) \mathbf{N}+\tau f_{t} \mathbf{B},
\end{gathered}
$$

and

$$
\mathbf{x}_{t t}=f_{t t} \mathbf{N}
$$

we obtain the desired results.

\section{Curvature of a surface defined by a homogeneous normal sectional parametrization}

In this section, we assume that both $f$ and $g$ are functions of a single variable $t$, that is, $f(s, t)=f(t)$ and $g(s, t)=g(t)$. Although we use ordinary differentiation under these assumptions, we still use the notations $f_{t}$ of partial differential, instead of the ordinary differential $f^{\prime}(t)$. Then, $f_{s}=0$ and $g_{s}=0$, and so we have $S=0$. Thus, the results given in Section 2 are simply expressed.

Definition 3.1. Let $\gamma$ be a regular curve with a unit speed in $\mathbb{R}^{3}$. If the parametrization $\mathbf{x}$ is defined by

$$
\mathbf{x}(s, t)=\gamma(s)+f(t) \mathbf{N}(s)+g(t) \mathbf{B}(s),
$$

that is, $f(s, t)=f(t)$ and $g(s, t)=g(t)$ are both single variable functions, then we call the parametrization $\mathbf{x}$ by the homogeneous normal sectional parametrization of $\gamma$.

REMARK 3.2. Let $\mathbf{x}$ be a homogeneous normal sectional parametrization of $\gamma$ with $f(s, t)=f(t)$ and $g(s, t)=g(t)$. Then $\mathbf{x}_{s}=R \mathbf{T}-\tau g \mathbf{N}+$ $\tau f \mathbf{B}$ and $\mathbf{x}_{t}=f_{t} \mathbf{N}+g_{t} \mathbf{B}$. Also, we have

(1) $\mathbf{x}_{s} \times \mathbf{x}_{t}=-\tau\left(f f_{t}+g g_{t}\right) \mathbf{T}-R g_{t} \mathbf{N}+R f_{t} \mathbf{B}$, and so

$$
g=\tau^{2}\left(f f_{t}+g g_{t}\right)^{2}+R^{2}\left(f_{t}^{2}+g_{t}^{2}\right) .
$$

(2) $E=R^{2}+\tau^{2}\left(f^{2}+g^{2}\right)$.

(3) $F=\tau\left(f g_{t}-g f_{t}\right)$.

(4) $G=f_{t}^{2}+g_{t}^{2}$. 
Moreover, if $\gamma$ is a plane curve, then the above equations imply that

(1) $g=R^{2}\left(f_{t}^{2}+g_{t}^{2}\right)$.

(2) $E=R^{2}$.

(3) $F=0$.

(4) $G=f_{t}^{2}+g_{t}^{2}$.

We assume that $R \neq 0$ and $f_{t} g_{t} \neq 0$. Then $\mathbf{x}_{s} \times \mathbf{x}_{t} \neq O$, and so the parametrization $\mathbf{x}$ satisfies the regularity condition for surface.

TheOREM 3.3. Assume that $\gamma$ is a unit speed regular curve in $\mathbb{R}^{3}$. Let $p$ be a point in the image $\operatorname{Im}(\mathbf{x})$ of the homogeneous normal sectional parametrization $\mathbf{x}$ defined by

$$
\mathbf{x}(s, t)=\gamma(s)+f(t) \mathbf{N}(s)+g(t) \mathbf{B}(s)
$$

on some open rectangle $I \times J$. Then the Gaussian curvature $K$ at $p$ is

$$
\begin{aligned}
& K=\frac{R^{2}}{g^{2}}\left[-\kappa g_{t} P R+P\left(f g_{t} \tau^{2}-g f_{t} \tau^{2}+g g_{t} \tau_{s}+f f_{t} \tau_{s}\right)-\left(f_{t}^{2}+g_{t}^{2}\right)^{2} \tau^{2}\right] \\
+ & \frac{\tau\left(f f_{t}+g g_{t}\right)}{g^{2}}\left[P R\left(\kappa_{s} f-\kappa \tau g\right)-\kappa^{2} \tau\left(f f_{t}+g g_{t}\right) f_{t}^{2}-2 \kappa \tau f_{t}\left(f_{t}^{2}+g_{t}^{2}\right) R\right],
\end{aligned}
$$

where $P$ denotes $f_{t} g_{t t}-g_{t} f_{t t}$.

Proof. By the straightforward computations, we have that

(1) $\mathbf{x}_{s s}=-\left(\kappa_{s} f-\kappa \tau g\right) \mathbf{T}+\left(\kappa R-\tau_{s} g-\tau^{2} f\right) \mathbf{N}+\left(\tau_{s} f-\tau^{2} g\right) \mathbf{B}$.

(2) $\mathbf{x}_{s t}=-\kappa f_{t} \mathbf{T}+\left(-\tau g_{t}\right) \mathbf{N}+\left(\tau f_{t}\right) \mathbf{B}$.

(3) $\mathbf{x}_{t t}=f_{t t} \mathbf{N}+g_{t t} \mathbf{B}$.

Also, we obtain that

$L=\frac{1}{\sqrt{g}}\left[\tau\left(f f_{t}+g g_{t}\right)\left(\kappa_{s} f-\kappa \tau g\right)-R g_{t}\left(\kappa R-\tau_{s} g-\tau^{2} f\right)+R f_{t}\left(\tau_{s} f-\tau^{2} g\right)\right]$,

$$
M=\frac{\tau}{\sqrt{g}}\left[\kappa f_{t}\left(f f_{t}+g g_{t}\right)+R\left(f_{t}^{2}+g_{t}^{2}\right)\right],
$$

and

$$
N=\frac{R}{\sqrt{g}}\left(f_{t} g_{t t}-g_{t} f_{t t}\right)=\frac{R P}{\sqrt{g}} .
$$

Therefore, we have

$$
\begin{aligned}
& L N-M^{2} \\
& =\frac{R^{2}}{g}\left[-\kappa g_{t} P R+P\left(f g_{t} \tau^{2}-g f_{t} \tau^{2}+g g_{t} \tau_{s}+f f_{t} \tau_{s}\right)-\left(f_{t}^{2}+g_{t}^{2}\right)^{2} \tau^{2}\right] \\
& +\frac{\tau\left(f f_{t}+g g_{t}\right)}{g}\left[P R\left(\kappa_{s} f-\kappa \tau g\right)-\kappa^{2} \tau\left(f f_{t}+g g_{t}\right) f_{t}^{2}-2 \kappa \tau f_{t}\left(f_{t}^{2}+g_{t}^{2}\right) R\right] .
\end{aligned}
$$


Since $K=\frac{L N-M^{2}}{g}$, we obtain the desired result.

Corollary 3.4. (1) If $\gamma$ is a straight line in Theorem 3.3, then the Gaussian curvature is constant zero, namely, $K(p)=0$, for every point $p$.

(2) If $\gamma$ is a plane curve in Theorem 3.3, then

$$
K(p)=\frac{-\kappa g_{t}\left(f_{t} g_{t t}-g_{t} f_{t t}\right)}{R\left(f_{t}^{2}+g_{t}^{2}\right)^{2}} .
$$

REMARK 3.5. If $\gamma$ is a plane curve, $f(s, t)=f(t)$ and $g(s, t)=t$, then the above corollary implies that

$$
K(p)=\frac{\kappa f_{t t}}{R\left(f_{t}^{2}+1\right)^{2}} .
$$

Hence, if $R>0$, that is, $1>\kappa f$, then the sign of Gaussian curvature of the surface defined by the homogeneous normal sectional parametrization of a curve $\gamma$ is determined by the sign of $f_{t t}$. Thus, if a smooth function $f$ has a convex downward (or upward) graph on an open interval $J$, then $f_{t t}>0$ (or $f_{t t}<0$ ), and so the Gaussian curvature is always nonnegative (or nonpositive).

For example, let $\gamma$ be a circle of radius $r$ and $0<a<r$. Then the torus defined by $f(t)=\sqrt{a^{2}-t^{2}}$ and $g(t)=t$ in the parametrization $\mathbf{x}$ has the Gaussian curvature at $p \in \operatorname{Im}(\mathbf{x})$ as follows:

$$
K(p)=\frac{-\sqrt{a^{2}-t^{2}}}{a^{2}\left(r-\sqrt{a^{2}-t^{2}}\right)} .
$$

The following example is the case that $f(s, t)=t$ and $g(s, t)=g(t)$ :

EXAMPLE 3.6. Let $\gamma$ be a circle of radius $r$ and $0<a<r$. Then, the curvature $\kappa(s)=\frac{1}{r}$ and $\tau(s)=0$ for $\gamma(s)$, and hence, Theorem 3.3 implies that the torus defined by using $f(t)=t$ and $g(t)=\sqrt{a^{2}-t^{2}}$ in the parametrization $\mathbf{x}$ in Theorem 3.3 has the Gaussian curvature at $p \in \operatorname{Im}(\mathbf{x})$ as follows:

$$
K(p)=\frac{t}{a^{2}(t-r)}
$$

\section{References}

[1] Y. Chai and D. Lee Tubes in singular spaces of nonpositive curvature, J. Korean Math. Soc. 43 (2006), 1129-1142.

[2] Y. Chai and D. Lee A lower bound for area of compact singular surfaces of nonpositive curvature, Bull. Korean Math. Soc. 44 (2007), 103-108. 
[3] B. Y. Chen, When does the position vector of a space curve always lie in its rectifying plane, Am. Math. Mon. 110 (2003), 147-152.

[4] H. Liu, Curves in the lightlike cone, Contrib. Algebr. Geom. 45, 291-303 (2004). Erratum: 54, 469-470 (2013).

[5] H. Liu and Q. Meng, Representation formulas of curves in a 2 and 3 dimensional lightlike cone, Results Math. 59, 437-451 (2011). Erratum: 61, 423-424 (2012).

6] A. Pressley, Elementary Differential Geometry, Springer-Verlag, (2001).

[7] V. A. Toponogov, Differential Geometry of Curves and Surfaces, Birkhauser, (2006).

$*$

College of Global General Education

Gachon University

Sungnam 461-701, Republic of Korea

E-mail: dhl221@gachon.ac.kr

$* *$

Department of Mathematics

Kwangwoon University

Seoul 139-701, Republic of Korea

E-mail: hsyi@kw.ac.kr 